\title{
Continued nucleic acid tests for SARS-CoV-2 following discharge of patients with COVID-19 in Lu'an, China
}

\author{
Yong Lyu ${ }^{\text {Corresp., } 1}$, Danni Wang ${ }^{2}$, Xiude $\mathrm{Li}^{1,3}{ }^{1,}$, Tianqi Gong ${ }^{1}$, Pengpeng Xu ${ }^{1}$, Lei Liu ${ }^{1}$, Jie Sun ${ }^{1}$ \\ 1 Lu'an Municipal Center for Disease Control and Prevention, Lu'an, China \\ 2 Teaching Center for Preventive Medicine, School of Public Health, Anhui Medical University, Hefei, China \\ 3 Department of Nutrition and Food Hygiene, School of Public Health, Anhui Medical University, Anhui Hefei, 230032, China, Anhui Medical University, \\ Hefei, China \\ Corresponding Author: Yong Lyu \\ Email address: lyong@lacdc.com.cn
}

Background. Studies have shown that discharged Coronavirus disease 2019 (COVID-19) patients have retested positive for SARS-CoV-2 during a follow-up RT-PCR test. We sought to assess the results of continued nucleic acid testing for SARS-CoV-2 patients in COVID-19 patients after they were discharged in Lu'an, China.

Methods. We conducted RT-PCR tests on sputum, throat swabs, fecal or anal swabs, and urine samples collected from 67 COVID-19 patients following discharge. Samples were collected on the 7th and 14th days following discharge. Patients testing positive on the 7th or 14th day were retested after 24 hours until they tested negative twice.

Results. Seventeen $(17 / 67,25.4 \%)$ discharged COVID-19 patients had a positive RT-PCR retest for SARSCoV-2. Among them, 14 (82.4\%) were sputum positive, five (29.4\%) were throat swab positive, seven $(41.2 \%)$ were fecal or anal swab positive, one (5.9\%) was urine sample positive, five $(29.4 \%)$ were both sputum and throat swab positive, four (23.5\%) were both sputum and fecal test positive, and one $(5.9 \%)$ was positive of all four specimens. The shortest period of time between discharge and the last positive test was 7 days, the longest was 48 days, and the median was 16 days. The proportion of positive fecal or anal swab tests increased from the third week. The median Cq cut-off values after onset were 26.7 after the first week, 37.7 the second to sixth week, and 40 after the sixth week. There were no significant differences between the RT-PCR retest positive group and the unrecovered positive group.

Conclusions. There was a high proportion of patients who retested positive for COVID-19. Discharge criteria have remained fairly consistent so we encourage regions affected by COVID-19 to appropriately amend their current criteria. 
1 Continued nucleic acid tests for SARS-CoV-2 following

2 discharge of patients with COVID-19 in Lu'an, China

3

4

5

6

\section{7}

Yong Lyu ${ }^{1}$, Danni Wang ${ }^{2}$, Xiude $\mathrm{Li}^{1,3}$, Tianqi Gong ${ }^{1}$, Pengpeng $\mathrm{Xu}^{1}$, Lei Liu ${ }^{1}$, Jie Sun ${ }^{1}$

Yong Lyu and Danni Wang are contributed equally to this article.

${ }^{1}$ Lu'an Municipal Center for Disease Control and Prevention, Anhui Lu'an, China

${ }^{2}$ Teaching Center for Preventive Medicine, School of Public Health, Anhui Medical University, Anhui Hefei, China

${ }^{3}$ Department of Nutrition and Food Hygiene, School of Public Health, Anhui Medical University, Anhui Hefei, China

\section{Corresponding Author:}

Yong Lyu ${ }^{1}$

Lu'an Municipal Center for Disease Control and Prevention, Gaocheng Road, Lu'an 237001, China

Email address: lyong@lacdc.com.cn

\section{Abstract}

Background. Studies have shown that discharged Coronavirus disease 2019 (COVID-19) patients have retested positive for SARS-CoV-2 during a follow-up RT-PCR test. We sought to assess the results of continued nucleic acid testing for SARS-CoV-2 patients in COVID-19 patients after they were discharged in Lu'an, China.

Methods. We conducted RT-PCR tests on sputum, throat swabs, fecal or anal swabs, and urine samples collected from 67 COVID-19 patients following discharge. Samples were collected on the 7th and 14th days following discharge. Patients testing positive on the 7th or 14th day were retested after 24 hours until they tested negative twice.

Results. Seventeen (17/67, 25.4\%) discharged COVID-19 patients had a positive RT-PCR retest for SARS-CoV-2. Among them, 14 (82.4\%) were sputum positive, five (29.4\%) were throat swab positive, seven $(41.2 \%)$ were fecal or anal swab positive, one $(5.9 \%)$ was urine sample positive, five (29.4\%) were both sputum and throat swab positive, four (23.5\%) were both sputum and fecal test positive, and one (5.9\%) was positive of all four specimens. The shortest period of time between discharge and the last positive test was 7 days, the longest was 48 days, and the median was 16 days. The proportion of positive fecal or anal swab tests increased from the third week. The median Cq cut-off values after onset were 26.7 after the first week, 37.7 the second to sixth week, and 40 after the sixth week. There were no significant differences between the RT-PCR retest positive group and the unrecovered positive group. 
38 Conclusions. There was a high proportion of patients who retested positive for COVID-19.

39

40

41

42

43

44

45

46

47

48

49

50

51

52

53

54

55

56

57

58

59

60

61

62

63

64

65

66

67

68

69

70

71

72

73

74

75

76

Discharge criteria have remained fairly consistent so we encourage regions affected by COVID19 to appropriately amend their current criteria.

\section{Introduction}

Coronavirus disease 2019 (COVID-19), which is caused by the novel severe acute respiratory syndrome coronavirus 2 (SARS-CoV-2), has spread rapidly since December 2019 (Li et al., 2020b; Zhou et al., 2020b). There have been a total of 85,557 confirmed COVID-19 cases in mainland China as of October 10, 2020. 94.3\% (80,705/85,558) of these cases recovered and were discharged from the hospital (National Health Commission of the People's Republic of China, 2020). The high recovery rate improved public confidence in the government's response to the emergency, however, the situation around the world remains grim. As of October 152020 , there have been 38,202,956 confirmed cases of COVID-19, including 1,087,069 deaths, reported to the World Health Organization (WHO), indicating that this pandemic cannot be ignored, especially in this era of globalization (World Health Organization,2020. https://covid19. who.int).

COVID-19 patients discharged in China were RT-PCR tested and found to be positive for SARS-CoV-2 again during follow-up care (China News Weekly; Dou et al., 2020; Lan et al., 2020; Li et al., 2020a; Tang et al., 2020; Xiao et al., 2020; Xing et al., 2020; Zhang et al., 2020; Zheng et al., 2020). Other countries have also reported patients that were positive when retested (Abdullah et al., 2020; Cento et al., 2020; Hartman et al., 2020; Zanardini et al., 2020). These studies have not shown that patients who retested positively were contagious (Wang, 2020), however, it has created public concern.

The World Health Organization (WHO) has updated its criteria for discharging isolated COVID-19 patients; in this criteria retesting is not required (World Health Organization, 2020. https://www.who.int/news-room/commentaries/detail/criteria-for-releasing-covid-19-patientsfrom-isolation; World Health Organization, 2021. https://www.who.int/publications/i/item/clinicalmanagement-of-covid-19). Countries have also updated their discharge protocols. For example, according to the new Korea Centers for Disease Control (KCDC) discharge protocols, "no additional tests are required for cases that have been discharged from isolation" (Korea Centers for Disease Control(KCDC), 2020). However, there is merit to retesting discharged cases and additional study is required to re-evaluate the current discharge criteria. We present the results of continued nucleic acid testing following the discharge of patients with COVID-19 in Lu'an, China.

\section{Materials \& Methods}

\section{Setting and samples}

There have been a total of 69 confirmed COVID-19 cases in Lu'an, China, as of April 16, 2020, with the first case confirmed on January 22, 2020. The first case was discharged from the hospital on January 30, 2020 and the last case was discharged on March 3, 2020. Two COVID-

PeerJ reviewing PDF | (2020:08:51719:2:1:NEW 28 Apr 2021) 
7719 cases left Lu'an after being discharged from the hospital. Therefore, nucleic acid tests were 78 conducted for 67 cases.

79 Discharge criteria

80 The Chinese discharge criteria for COVID-19 patients have been consistent between the second 81 and the seventh iterations and are based on following criteria: 1) normal temperature $\left(<37.3{ }^{\circ} \mathrm{C}\right)$ 82 for 3 days; 2) reduced respiratory tract symptoms and clear clinical improvement confirmed by 83 pulmonary imaging, and 3) collection of two consecutive negative RT-PCR tests on respiratory 84 samples at least $24 \mathrm{~h}$ apart (National Health Commission of the People's Republic of China, 85 2020).

86 Sample collection methods

87 An epidemiological investigation was conducted and all 67 patients were confirmed to have 88 maintained distance from others and remained indoors after discharge, thus ruling out possible 89 reinfection. Sputum, throat, fecal or anal swabs, and urine samples were collected on the 7th and 90 14th day following discharge. If a patient tested negative, a second test was performed more than 9124 hours after the first test until the patient was confirmed to be negative for two consecutive 92 tests. The patient was immediately required to continue the quarantine protocol in the hospital 93 for at least 14 days.

94 Nucleic acid test strategy

95 RT-PCR was used to conduct nucleic acid testing. The RT-PCR kit was manufactured by Sun 96 Yat-sen University Da'an Gene Co., Ltd., China (20203400063). The ROC curve was used to 97 determine the ORF1 $\mathrm{ab}$ and $\mathrm{N}$ reference values, which according to the test results of the clinical 98 samples resulted in $\mathrm{Cq}$ values equal to 40. Samples were determined to be negative when 99 amplification above 40 was detected. Samples with amplification below cycle 37 indicated that 100 the diagnosis was positive. Testing was repeated for samples with a Cq range of 37 - 40. A 101 repeated $\mathrm{Cq}$ value less than 40 with an amplification curve that had obvious fluctuations 102 indicated a positive result; otherwise the result was determined to be negative.

103 Case definition

104 A retested positive case was defined as one that was positive for SARS-CoV-2 in any specimen 105 when RT-PCR tested following discharge. Recovered patients who were considered negative 106 were those who remained negative from discharge to the end of the study period. Secondary 107 cases were the cases resulting from a secondary transmission.

108 Statistical analysis

109 Data were double-entered using EpiData software, version 3.1 (EpiData Association, Denmark) 110 and were analyzed using SPSS software version 11.0 (SPSS, Chicago, IL, USA). Pearson Chi111 square was used to analyze and count data in different groups. The $\mathrm{T}$ test was used to compare 112 the mean between groups. Tests were designed as two-tailed and an alpha value of $\mathrm{p}<0.01$ was 113 considered to be statistically significant.

114 Ethical aspects 
115 The research protocols used in this study were reviewed and approved by the Lu'an Municipal 116 Health and Family Planning Commission and written informed consent was obtained from all 117 research participants.

118

119

120

121

122

123

124

125

126

127

128

129

130

131

132

133

134

135

136

137

138

139

140

141

142

143

144

145

146

147

148

149

150

151

152

153

154

\section{Results}

\section{Specimen nucleic acid tests}

Seventeen (17/67, 25.4\%) discharged COVID-19 patients retested positive for SARS-CoV-2 using RT-PCR testing. Fourteen (82.4\%) were sputum positive, five $(29.4 \%)$ were throat swab positive, seven $(41.2 \%)$ were fecal or anal swab positive, one $(5.9 \%)$ was urine sample positive, five $(29.4 \%)$ were both sputum and throat swab positive, four $(23.5 \%)$ were both sputum and fecal swab positive, and one (5.9\%) was positive in all four specimens (Table 1$)$.

Among the 17 cases, $11(64.7 \%)$ tested positive more than once following discharge and six (35.3\%) tested positive only once. The shortest amount of time between discharge and the last positive test was 7 days, the longest was 48 days, and the median interval was 16 days (Table 1).

No significant differences $(p=0.226)$ were observed between the average number of days required for the continued collection of positive fecal or anal swab samples (19.0 \pm 10.2 days) and other specimens (14.2 \pm 5.5 days) following discharge, whereas the proportion of positive fecal or anal swab samples increased from the third week (Table 1, Fig. 1).

Serial $\mathrm{Cq}$ value time course for sputum samples collected from recovered positive patients on the day of illness (ORF)

Daily serial RT-PCR Cq values for the 17 recovered positive patients are shown in Fig. 2.

Sputum's median Cq value in the first week after onset was 26.7 (14.2 to 40), 37.7 (24.6 to 40) from weeks two to six, and 40 in the majority of cases after six weeks.

\section{Secondary cases}

A total of 135 close contacts were tested relating to the 17 positive cases. The results of RT-PCR revealed that there were zero positive cases among those contacts.

\section{Comparing recovered positive and unrecovered positive patient characteristics}

We collected data regarding age, gender, Wuhan exposure history, underlying medical conditions, disease status, disease course, hormone use, and sputum samples' Cq value in the early stages of the disease then performed statistical comparisons. No significant differences were noted between the groups (Table 2).

\section{Discussion}

Our data demonstrate that approximately a quarter of patients with COVID-19 who recovered were found to be positive again following discharge according to the current discharge criteria in China. Most were sputum positive, throat swab positive, and fecal or anal swab positive. One patient tested positive via urine sample and only one patient was positive with all four specimens. Positive fecal or anal swab results increased from the third week onward. A recent study published in Lancet indicated that the median and longest duration of viral shedding in 
155 COVID-19 survivors was 20 and 37 days, respectively (Zhou et al., 2020a). However, we found

156

157

158

159

160

161

162

163

164

165

166

167

168

169

170

171

172

173

174

175

176

177

178

179

180

181

182

183

184

185

186

187

188

189

190

191

192

193

194 that all of the COVID-19 cases had a positive retest less than one month from symptom onset to discharge. The discharged patients that continued to be RT-PCR positive may not have mounted an effective immune response and the infection detected by the fecal samples may require a longer time to clear (Ling et al., 2020). Our results support these findings. Therefore, it is important to assess the viral nucleic acid concentration in fecal or anal swab samples during recovery.

The seventh iteration of the COVID-19 diagnosis and treatment plan in China emphasizes the health management and isolation of patients following discharge. We identified criteria between positive patients who recovered and those who did not recover in order to aid the early screening process. However, no significant differences were noted among common epidemiological characteristics, the clinical characteristics, and the disease status, which is inconsistent with the findings of other relevant studies. Ling et al. (2021) showed that SARS-CoV-2 RNA-negative conversions are risk factors for delayed discharge from the hospital ( $>21$ days), suggesting that there may be different discharge criteria in different regions. Our results showed no significant differences between severe, mild cases, and common cases in patients who retested positively after discharge. However, a study by Weerahandi et al. (2021) demonstrated that patients with severe cases of COVID-19 may develop health conditions several weeks after discharge, indicating that preventive measures are needed after discharge to reduce the risk of transmission in severely ill patients. Previous studies have suggested that this may be associated with host cell immunity (Ling et al., 2020). No secondary cases (cases resulting from a secondary transmission) were reported, which may be associated with the isolation measures. The Cq value of the majority of recovered patients with COVID-19 who recovered but were positive following discharge was greater than 35 within three weeks after onset, which means that infectivity may decrease significantly. The continued positive test results indicated that the possibility of viral transmission was still present and the infectious mechanism of the retested positive patients remains unknown. Viral shedding may have included the infectious virus, which should be determined in future studies. Isolation practices have been improved for COVID-19 patients in China who retested positive for viral nucleic acids following discharge (The Central People's Government of the People's Republic of China, 2020). However, we suggest that these discharge criteria are insufficient. SARS-COV-2 is believed to be a highly adaptable virus and South Korea reported 51 patients in April 2020 who retested positive (People's Daily Overseas, 2020). To prevent SARS-CoV-2 transmission that may lead to new COVID-19 cases, it is essential that patients are not discharged from the hospital until they are no longer infectious. Therefore, we encourage vulnerable regions to appropriately improve their discharge criteria for COVID-19 patients. We suggest that the frequency of negative RT-PCR tests for patient samples and the time required from symptom onset or admission to discharge be increased. We also recommend expanded tests to include fecal or anal swab samples prior to discharge. As validated serology tests become available, the inclusion of tests for patient antibody levels may also be considered. If conditions permit, additional measures such as home isolation and discharge follow-up

PeerJ reviewing PDF | (2020:08:51719:2:1:NEW 28 Apr 2021) 
195

196

197

198

199

200

201

202

203

204

205

206

207

208

209

210

211

212

213

214

215

216

217

218

219

220

221

222

223

224

225

226

227

228

229

230

231

232

233

234

235

236

monitoring should be undertaken to ensure that no secondary cases occur. WHO's assessment suggests that COVID-19 can be characterized as a pandemic and it is predicted that more cases will occur globally in the future (World Health Organization, 2020. https://www.who.int/dg/speeches/detail/who-director-general-s-opening-remarks-at-the-mediabriefing-on-covid-19---11-march-2020). Our hope is that we can increase the preventive measures and control the infection of this virus in China and other areas by introducing revised discharge criteria.

Our study has some deficiencies and limitations. Firstly, due to the limitations of on-site investigations, we mainly used descriptive epidemiological investigation methods and, as a result, could not explore the specific mechanism for recovering positivity in discharged patients. Secondly, due to the limited sample size, we did not find a difference in the characteristics of recovered positive and unrecovered positive patients. Therefore, a larger cohort is needed to verify our conclusions. Thirdly, virus culture tests need to be performed in laboratories that reach biosafety level 3. As we did not have access to such resources, this will be our focus in future studies.

\section{Conclusions}

We confirm that there is a high probability of testing positive after COVID-19 patients are discharged. China's discharge criteria have remained fairly consistent so we encourage vulnerable regions to appropriately strengthen discharge criteria for COVID-19 patients, despite not being able to verify their infectivity. We hope that there will be more opportunities to conduct viral cell culture tests along with RT-PCR in biosafety level 3 laboratory conditions to better understand and verify the infectivity of COVID-19. This will help us better develop and improve the discharge criteria.

\section{Acknowledgements}

We wish to thank the Lu'an Municipal Health and Family Planning Commission, and Lu'an Municipal People's Hospital for their support and cooperation during our on-site investigation.

\section{References}

Abdullah MS, Chong PL, Asli R, Momin RN, Mani BI, Metussin D, and Chong VH. 2020. Post discharge positive re-tests in COVID-19: common but clinically non-significant. Infect Dis (Lond) 52:743-745. 10.1080/23744235.2020.1780309

Cento V, Colagrossi L, Nava A, Lamberti A, Senatore S, Travi G, Rossotti R, Vecchi M, Casati O, Matarazzo E, Bielli A, Casalicchio G, Antonello M, Renica S, Costabile V, Scaglione F, Fumagalli R, Ughi N, Epis OM, Puoti M, Vismara C, Faccini M, Fanti D, Alteri C, and Perno CF. 2020. Persistent positivity and fluctuations of SARS-CoV-2 RNA in clinicallyrecovered COVID-19 patients. J Infect 81:e90-e92. 10.1016/j.jinf.2020.06.024

China News Weekly. 14\% of discharged patients in Guangdong were positive for re examination. Experts: it may still be infectious. Available at http://www.inewsweek.cn/society/2020-03-05/8620.shtml (accessed 5, March 2020). 
237

238

239

240

241

242

243

244

245

246

247

248

249

250

251

252

253

254

255

256

257

258

259

260

261

262

263

264

265

266

267

268

269

270

271

272

273

274

275

276

277

278

279

280

281

282

283

284

285

286

Dou P, Zhang S, Wang C, Cai L, Liu Z, Xu Q, Li X, Meng Y, Rong Y, Li S, Hu C, and Xu K. 2020. Serial CT features in discharged COVID-19 patients with positive RT-PCR re-test. Eur J Radiol 127:109010. 10.1016/j.ejrad.2020.109010

Hartman WR, Hess AS, and Connor JP. 2020. Persistent viral RNA shedding after COVID-19 symptom resolution in older convalescent plasma donors. Transfusion. $10.1111 /$ trf. 15927

Korea Centers for Disease Control (KCDC). Findings from investigation and analysis of repositive cases. Available at https://www.cdc.go.kr/board/board.es?mid=a30402000000\&bid=0030\&act=view\&list_no $=367267 \& n P a g e=1$ (accessed 15, October 2020).

Lan L, Xu D, Ye G, Xia C, Wang S, Li Y, and Xu H. 2020. Positive RT-PCR Test Results in Patients Recovered From COVID-19. JAMA 323:1502-1503. 10.1001/jama.2020.2783

Li J, Long X, Fang X, Zhang Q, Hu S, Lin Z, and Xiong N. 2020a. SARS-CoV-2 positivity in a discharged COVID-19 patient: a case report. Clin Microbiol Infect 26:1115-1117. 10.1016/j.cmi.2020.04.032

Li Q, Guan X, Wu P, Wang X, Zhou L, Tong Y, Ren R, Leung KSM, Lau EHY, Wong JY, Xing X, Xiang N, Wu Y, Li C, Chen Q, Li D, Liu T, Zhao J, Liu M, Tu W, Chen C, Jin L, Yang R, Wang Q, Zhou S, Wang R, Liu H, Luo Y, Liu Y, Shao G, Li H, Tao Z, Yang Y, Deng Z, Liu B, Ma Z, Zhang Y, Shi G, Lam TTY, Wu JT, Gao GF, Cowling BJ, Yang B, Leung GM, and Feng Z. 2020b. Early Transmission Dynamics in Wuhan, China, of Novel Coronavirus-Infected Pneumonia. N Engl J Med 382:1199-1207. 10.1056/NEJMoa2001316

Lin P, Chen W, Huang H, Lin Y, Cai M, Lin D, Cai, Su Z, Zhuang X, Yu X. 2021. Delayed discharge is associated with higher complement $\mathrm{C} 3$ levels and a longer nucleic acidnegative conversion time in patients with COVID-19. Sci Rep. 11(1):1233.10.1038/s41598-021-81010-3.

Ling Y, Xu SB, Lin YX, Tian D, Zhu ZQ, Dai FH, Wu F, Song ZG, Huang W, Chen J, Hu BJ, Wang S, Mao EQ, Zhu L, Zhang WH, and Lu HZ. 2020. Persistence and clearance of viral RNA in 2019 novel coronavirus disease rehabilitation patients. Chin Med J (Engl) 133:1039-1043. 10.1097/CM9.0000000000000774

National Health Commission of the People's Republic of China. Diagnosis and treatment plan of COVID-19 (trial version seventh). Available at http://www.nhc.gov.cn/yzygj/s7653p/202003/46c9294a7dfe4cef80dc7f5912eb1989.shtml (accessed 4, March 2020).

National Health Commission of the People's Republic of China. Update on COVID-19. Available at $h$ ttp://www.nhc.gov.cn/xcs/yqtb/202010/b4298767b6104a80a15ec7e8e477e322.shtml (accessed 10, October 2020).

People's Daily Overseas. Read about the global epidemic. Available at http://news.haiwainet.cn/n/2020/0407/c3541093-31760857.html (accessed 9, April 2020).

Tang X, Zhao S, He D, Yang L, Wang MH, Li Y, Mei S, and Zou X. 2020. Positive RT-PCR tests among discharged COVID-19 patients in Shenzhen, China. Infect Control Hosp Epidemiol 41:1110-1112. 10.1017/ice.2020.134

The Central People's Government of the People's Republic of China. COVID-19 work plan of reexamination for discharged patients. Available at http://www.gov.cn/zhengce/content/2020-04/08/content_5500372.htm (accessed 10, April 2020).

Wang YXJ. 2020. CT suggests discharged Covid-19 patients who were retested RT-PCR positive again for SARS-CoV-2 more likely had false negative RT-PCR tests before discharging. Quant Imaging Med Surg 10:1396-1400. 10.21037/qims-2020-19

PeerJ reviewing PDF | (2020:08:51719:2:1:NEW 28 Apr 2021) 
287

288

289

290

291

292

293

294

295

296

297

298

299

300

301

302

303

304

305

306

307

308

309

310

311

312

313

314

315

316

317

318

319

320

321

322

323

324

325

326

327

328

329

Weerahandi H, Hochman KA, Simon E, Blaum C, Chodosh J, Duan E, Garry K, Kahan T, Karmen-Tuohy S, Karpel HC, Mendoza F, Prete AM, Quintana L, Rutishauser J, Martinez LS, Shah K, Sharma S, Simon E, Stirniman A, Horwitz LI. 2021. PostDischarge Health Status and Symptoms in Patients with Severe COVID-19. J. Gen. Int. Med. 1-8.10.1007/s11606-020-06338-4. Online ahead of print.

World Health Organization (WHO). Clinical management of COVID-19. Available at https://www.who.int/publications/i/item/clinical-management-of-covid-19 (accessed 25, January 2021).

World Health Organization (WHO). Coronavirus disease (COVID-19) Situation dashboard. Available at https://covid19. who.int/ (accessed 15, October 2020).

World Health Organization (WHO). Criteria for releasing COVID-19 patients from isolation. Available at https://www.who.int/news-room/commentaries/detail/criteria-for-releasingcovid-19-patients-from-isolation (accessed 15, October 2020).

World Health Organization (WHO). WHO Director-General's opening remarks at the media briefing on COVID-19 - 11 March 2020. Available at https://www.who.int/dg/speeches/detail/who-director-general-s-opening-remarks-at-themedia-briefing-on-covid-19---11-march-2020 (accessed 12, March 2020).

Xiao AT, Tong YX, and Zhang S. 2020. False negative of RT-PCR and prolonged nucleic acid conversion in COVID-19: Rather than recurrence. J Med Virol. 10.1002/jmv.25855

Xing Y, Mo P, Xiao Y, Zhao O, Zhang Y, and Wang F. 2020. Post-discharge surveillance and positive virus detection in two medical staff recovered from coronavirus disease 2019 (COVID-19), China, January to February 2020. Euro Surveill 25. 10.2807/15607917.ES.2020.25.10.2000191

Zanardini C, Saccani B, Franceschetti L, Zatti S, Sartori E, and Prefumo F. 2020. Retest positive for SARS-CoV-2 RNA of recovered pregnant women with COVID-19. Ultrasound Obstet Gynecol. 10.1002/uog.23144

Zhang B, Liu S, Dong Y, Zhang L, Zhong Q, Zou Y, and Zhang S. 2020. Positive rectal swabs in young patients recovered from coronavirus disease 2019 (COVID-19). J Infect 81:e49e52. 10.1016/j.jinf.2020.04.023

Zheng KI, Wang XB, Jin XH, Liu WY, Gao F, Chen YP, and Zheng MH. 2020. A Case Series of Recurrent Viral RNA Positivity in Recovered COVID-19 Chinese Patients. J Gen Intern Med 35:2205-2206. 10.1007/s11606-020-05822-1

Zhou F, Yu T, Du R, Fan G, Liu Y, Liu Z, Xiang J, Wang Y, Song B, Gu X, Guan L, Wei Y, Li H, Wu X, Xu J, Tu S, Zhang Y, Chen H, and Cao B. 2020a. Clinical course and risk factors for mortality of adult inpatients with COVID-19 in Wuhan, China: a retrospective cohort study. Lancet 395:1054-1062. 10.1016/S0140-6736(20)30566-3

Zhou P, Yang XL, Wang XG, Hu B, Zhang L, Zhang W, Si HR, Zhu Y, Li B, Huang CL, Chen $H D$, Chen J, Luo Y, Guo H, Jiang RD, Liu MQ, Chen Y, Shen XR, Wang X, Zheng XS, Zhao K, Chen QJ, Deng F, Liu LL, Yan B, Zhan FX, Wang YY, Xiao GF, and Shi ZL. 2020 b. A pneumonia outbreak associated with a new coronavirus of probable bat origin. Nature 579:270-273. 10.1038/s41586-020-2012-7

PeerJ reviewing PDF | (2020:08:51719:2:1:NEW 28 Apr 2021) 


\section{Table $\mathbf{1}$ (on next page)}

Table 1. Results of nucleic acid tests for 17 discharged COVID-19 cases who were RTPCR retested positive again for SARS-CoV-2 


\section{Table 1}

2 Results of nucleic acid tests for 17 discharged COVID-19 cases who were RT-PCR retested positive again for 3 SARS-CoV-2

\begin{tabular}{lrr}
\hline Variables & Number (N=17) & Days \\
\hline Positive of specimens & $14(82.4 \%)$ & \\
Sputum & $7(41.2 \%)$ & \\
Fecal or swab & $5(29.4 \%)$ & \\
Throat & $1(5.9 \%)$ & \\
Urine & $5(29.4 \%)$ & \\
Both sputum and throat swab & $4(23.5 \%)$ & \\
Both sputum and fecal & $1(5.9 \%)$ & \\
All four specimens & $6(35.3 \%)$ & \\
Frequency of positive (times) & $11(64.7 \%)$ & \\
1 & & \\
$>1$ & & 16 \\
Days of discharge to the last positive (days) & & $14.2 \pm 5.5$ \\
Shortest & & \\
Longest & & \\
$\quad$ Median & & \\
Days for collection of continuous positive (days) & & \\
Fecal or anal swab samples & & \\
Others & & \\
\hline
\end{tabular}

4 


\section{Table 2 (on next page)}

Table 2 Comparing recovered positive and unrecovered positive patient characteristics 


\section{Table 2}

2 Comparing recovered positive and unrecovered positive patient characteristics

\begin{tabular}{|c|c|c|c|}
\hline Variable & Recovered positive patients & Unrecovered positive patients & $p$ value \\
\hline Age (years) & $42(3$ to 66$)$ & $40.5(1$ to 78$)$ & 0.954 \\
\hline Gender & & & 0.958 \\
\hline Male $[\mathrm{n},(\%)]$ & $11(25.6 \%)$ & $32(74.4 \%)$ & \\
\hline Female $[\mathrm{n},(\%)]$ & $6(25.0 \%)$ & $18(75.0 \%)$ & \\
\hline Wuhan exposure history $[\mathrm{n},(\%)]$ & $3(17.6 \%)$ & $13(26.0 \%)$ & 0.712 \\
\hline Underlying medical conditions $[\mathrm{n},(\%)]$ & $3(17.6 \%)$ & $2(4.0 \%)$ & 0.188 \\
\hline Disease status & & & 0.317 \\
\hline Mild and common cases $[\mathrm{n},(\%)]$ & $45(90.0 \%)$ & $5(10.05)$ & \\
\hline Severe cases $[\mathrm{n},(\%)]$ & $13(76.5 \%)$ & $4(23.5 \%)$ & \\
\hline Disease course (days) & $22.5 \pm 5.8$ & $22.7 \pm 6.8$ & 0.901 \\
\hline Hormone use $[\mathrm{n},(\%)]$ & $3(17.6 \%)$ & $14(28.0 \%)$ & 0.600 \\
\hline Cq value of sputum in the initial stage of the & $29.4(14.2$ to 40$)$ & $28.8(12.7$ to 40$)$ & 0.792 \\
\hline
\end{tabular}

3 


\section{Figure 1}

Fig. 1. Positive percentage of different types of specimens following discharge

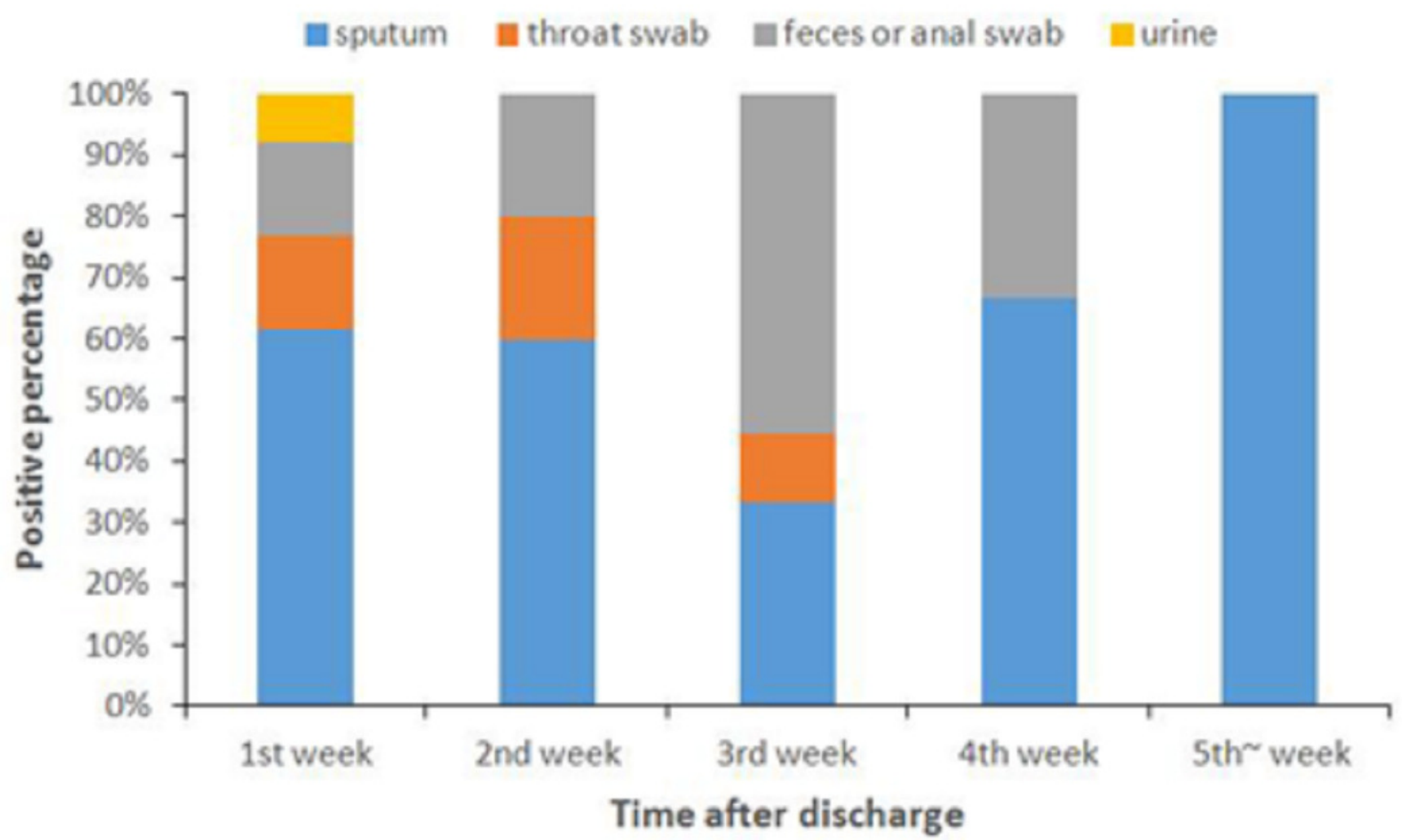


Figure 2

Fig. 2. Serial $\mathrm{Cq}$ value time course for sputum samples collected from recovered positive patients on the day of illness (ORF)

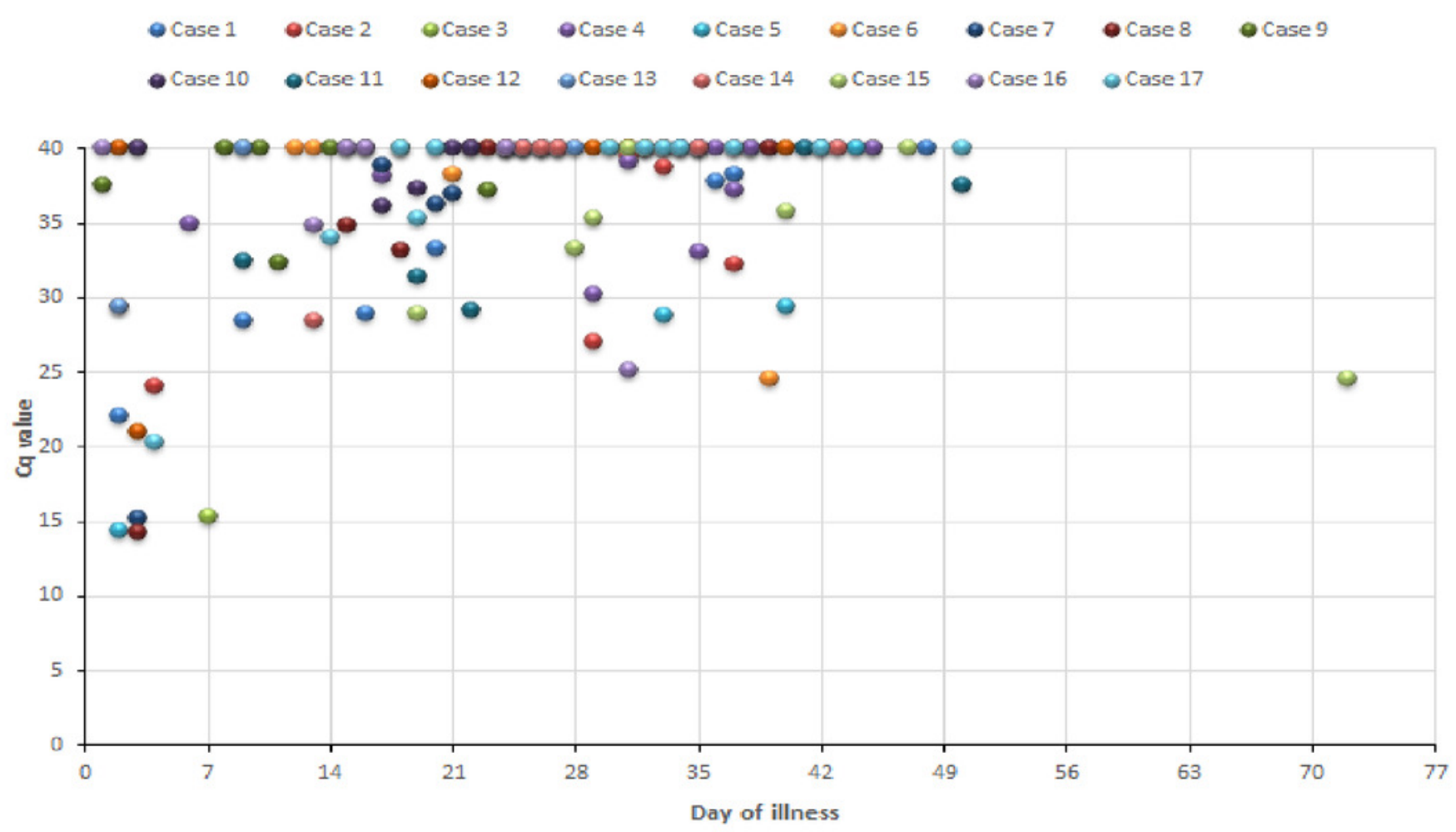

